\title{
Superspreaders help covid-19 elimination
}

\author{
David Kault, adjunct snr lect, maths, James Cook University \\ and General Practitioner
}

May 8, 2020

\section{Abstract}

There seems to be widespread pessimism regarding the ability of a nation to eliminate covid. One factor in this pessimism seems to be concern that covid might always be able to re-emerge because of the ongoing presence of unrecognised asymptomatic cases. However, it is shown here that it should be possible to eliminate covid more easily than anticipated, for a reason that at first glance seems paradoxical - the presence of superspreaders. If superspreaders are responsible for most of the spread, then, with the average number of secondary cases fixed at say $R_{0}=2.5$, we have to conclude that superspreaders are relatively rare. When towards the end of an elimination program, there are very few infected people, whether symptomatic or asymptomatic, that small number of people may well not include any superspreaders. As a result, chance effects may make extinction likely. Nevertheless it is clear an attempt at elimination will require a rather onerous "lockdown". In this paper we use a branching processes model to look at the tradeoff between risk of disease re-emergence and the length of "lockdown" required after a program of elimination has dropped the number of symptomatic cases in a region to just one. 


\section{Introduction}

The experience of Taiwan, China, Vietnam, New Zealand and perhaps Australia indicates that covid-19 can be eliminated. Elimination may require lowering the reproductive rate of the infection by "lockdown" measures until there are very few active cases and then perhaps waiting for several incubation periods without new cases before ending the lockdown.

When numbers are large, each generation of cases will increase by a factor of $\approx R_{0}$ (ignoring saturation effects). However, when numbers are very low, stochastic effects are important, particularly if superspreaders are prominent. To take an extreme example, it is clear that if superspreaders were all important, so that the average $R_{0}$ of say 2.5 was made up of $1 \%$ of infected people, who each spread the disease to 250 others and $99 \%$ who spread the disease to no-one, then there is at least a $99 \%$ chance that any randomly chosen infected person will not generate ongoing transmission. In general, a degree of superspreading, seems to be important across a range of infectious disease. ${ }^{[1,2]}$ A negative binomial distribution seems to approximate the distribution of the number of secondary cases generated by a primary case of an infectious disease. ${ }^{[1,2]}$ This distribution depends on the average number infected $R_{0}$ and a dispersion parameter $r$ (some authors use $k$ in place of $r$ ). With $R_{0}$ fixed, if $r \rightarrow \infty$, there is no superspreading phenomenon and the number of secondary cases is the total randomness described by a Poisson distribution. If $r \rightarrow 0$, superspreading becomes increasingly important, with a smaller and smaller proportion of infectious cases spreading the disease to more and more people. Likewise, as $r \rightarrow 0$, if we start with a randomly chosen infected person, the chance that they are a superspreader becomes smaller and so the chance that they give rise to ongoing disease transmission reduces. Correspondingly, with very low numbers of infected people close to the end of a program of elimination, as $r \rightarrow 0$, the probability of elimination becomes high.

There are various estimates of the dispersion parameter $r$ for the distribution of secondary covid cases that have occurred from various primary cases. For the distribution before lockdown there is an estimate that $r=0.1$ from observation of the initiation of the epidemic in various countries. ${ }^{[3]} \mathrm{A}$ paper based on data from the early epidemic in China, states that there will be a probability of $<50 \%$ of covid elimination if there are at least 4 cases 
present. ${ }^{[4]}$ It is difficult to find the direct estimate of $r$ in this paper but this probability of elimination is compatible with an $r$ value of between 0.126 and 0.172. A paper based on data after lockdown in China states that $80 \%$ of cases are caused by $8.9 \%$ of infected people. ${ }^{[5]}$ With $R_{0}$ also specified, it is not possible to precisely match the two figures $80 \%$ and $8.9 \%$ by varying the single parameter $r$, though $r=0.126$ provides a close approximation. (Unfortunately, the paper directly states a negative binomial dispersion parameter value of 0.58 , but this is not compatible with their data.) The parameter $r$ in the case of SARS is estimated to be $0.16-0.17^{[2]} \mathrm{A}$ million fold variation in viral load in the secretions of covid patients also suggests a prominent role for superspreaders. ${ }^{[6]}$ Given these various estimates of $r$, it seems that the true distribution of the number of secondary cases of covid generated by various primary cases, can be approximated by a negative binomial distribution with dispersion parameter $r$ in the range 0.10 to 0.17 . Accordingly, two negative binomial distributions, one with $r=0.10$ and the other with $r=0.17$, are used in a model of the last stage of a program of regional covid elimination.

For both distributions, the case of a policy of release of lockdown once there is just a single newly diagnosed symptomatic case, is examined. Results are obtained by analytical methods. The effect of two further policies are obtained by simulation. These policies are waiting one incubation period after this time before lifting restrictions and waiting two incubation periods before lifting restrictions.

This paper assumes that an attempt has been made to eliminate covid-19 from a region/province/nation. It assumes no appreciable herd immunity. It also assumes that there is no quarantine free cross border human travel into the region considered and this restriction will be maintained. We make the conservative assumption that the moment lockdown is lifted, transmission immediately goes back to its pattern at the beginning of the epidemic, which for both distributions is assumed to have $R_{0}=2.5$.

It is known that a proportion of cases are asymptomatic. Here this proportion $p_{a}$, is taken to be $50 \%$. It is further assumed that the symptomatic cases up to their time of isolation and the asymptomatic cases, both have the same distribution for the number of secondary infections they generate. This too seems likely to be a conservative assumption. 


\section{Methods}

It is known from the theory of branching processes in discrete time, that the probability of extinction of a branching process is given by the (smallest) solution to the equation $\phi(u)=u$ where $\phi(x)$ is the probability generating function for the number of secondary cases of a single index case. ${ }^{[7]}$ This equation can be solved by Newton's method. If the distribution of secondary cases is negative binomial with $R_{0}=2.5$ and $r=0.10$, the probability of extinction of a single branching process is 0.861 . With the same $R_{0}$ and $r=0.17$ the probability of extinction is 0.784 . We denote these probabilities of extinction of a line from a single case as $p_{e}$.

However, once a closed region is down to a single newly diagnosed symptomatic case, we are assuming that there will be on average one unnoticed asymptomatic infectious person $\left(p_{a}=50 \%\right)$. It is assumed, as an uninformative Bayesian prior, that the actual number of asymptomatic cases will follow a geometric distribution. Nevertheless, as described above, there may be no ongoing transmission from any one case and so it is possible that there will be no ongoing transmission from however many infectious people there are left. We make two conservative assumptions here. First, that the last symptomatic case has already definitely seeded one other case before diagnosis and isolation. Second, that with lockdown lifted as soon as the number of newly diagnosed symptomatic cases is down to $1, R_{0}$ for all infectious people still present, immediately goes back to 2.5. It is shown in Attachment 1 that the probability of epidemic re-emergence will then be $\frac{\left(1-p_{a}\right) p_{e}}{1-p_{a} p_{e}}$. We denote this probability as $p_{T 0}$ where " $T$ " denotes Total disease elimination and the " 0 " stands for release of lockdown zero incubation periods after the the number of newly diagnosed symptomatic cases has dropped to one.

There are two further policy scenarios considered - continuing restrictions for 1 incubation period after the last symptomatic person has been isolated, and continuing retrictions for 2 incubation periods. During this period of ongoing lockdown it is assumed that a negative binomial distribution of secondary cases will still apply but with $R_{0}=0.8$. However, the dispersion parameter $r$ is assumed to be unchanged. The probability that the disease 
will truly have been eliminated if no further symptomatic case emerges during this extended lockdown is calculated by simulation. The probability of total elimination of the disease after 1 and after 2 incubation periods is denoted $p_{T 1}$ and $p_{T 2}$ respectively. Each simulation employs 10 million runs. A fortran program corresponding to these simulations is given as an attachment.

\section{Results}

Table : Results with $p_{e}$ and $p_{T 0}$ calculated analytically and $p_{T 1} \& p_{T 2}$ calculated by simulation. The analytic calculation assumes that the last diagnosed case has already seeded exactly one other case before isolation and then $R_{0}$ immediately returns to 2.5. The simulations assume that during isolation, all infected people seed cases according to a distribution with $R_{0}=0.8$, but afterwards $R_{0}$ returns immediately to 2.5

\begin{tabular}{ccccc}
\hline$r$ & $p_{e}$ & $p_{T 0}$ & $p_{T 1}$ & $p_{T 2}$ \\
\hline 0.10 & 0.861 & 0.755 & 0.974 & 0.9978 \\
0.17 & 0.784 & 0.645 & 0.961 & 0.9966 \\
\hline
\end{tabular}

Unfortunately, in implementing policies whose outcomes are denoted $p_{T 1}$ and $p_{T 2}$, there will be occasions where relief from "lockdown" will be further delayed by re-emergence of symptomatic cases before the one or two incubation periods are completed. In the case of both the distributions, this happens about $31 \%$ and $34 \%$ of the time with a policy of a 1 or 2 incubation wait respectively.

\section{Discussion}

A policy of elimination requires a more intense "lockdown" than a policy of "flattening the curve" so that the epidemic peak is within the capacity of a region's ICU beds. However, a study by the Imperial College, ${ }^{[8]}$ shows that there are two rather fine lines between flattening the curve sufficiently and on the one hand, elimination, or on the other hand, an insufficiently flattened epidemic and a huge death rate. Even if "optimal" curve flattening can be achieved there will still be many deaths. In addition, whilst slightly less intense lockdown is required for curve flattening rather than elimination, the 
period of "lockdown" for curve flattening, will have to be continued for far longer. An advantage of the curve flattening approach is that curve flattening could lead to herd immunity and thus allow less restricted international travel to parts of the world that have not achieved elimination, though this prospect is uncertain. On the other hand, international travel without quarantine will not be possible between a country that has achieved elimination and a country which has not, at least until a vaccine is available. For this author, the arguments in favour of a policy of elimination are ovewhelming, for nations/regions which have the resources to implement it and who have secure borders. New Zealand is following a policy of elimination and there seems to be emerging realisation that this is a real option for Australia. Since the intensity of "lockdown" required for elimination will be quite onerous, it is important that the length of "lockdown" required is balanced against the probability that this lockdown period will be successful in eliminating the virus.

The calculations show that there is better than $99.7 \%$ chance of elimination if two incubation periods pass without re-emergence of symptomatic cases. It would seem that, when numbers of symptomatic cases have been reduced to rather low levels, lockdown periods, could be decreased further by subdividing a region. A region implementing an elimination strategy could be subdivided into subregions which could be temporarily disconnected from each other in terms of human travel, such that each subregion has only one case. Relief from severe restrictions could then commence quite quickly.

There are some clear limitations to this study. No sensitivity analysis has been done beyond calculations for the two specific distributions described. In particular, there is no sensitivity analysis for likely different values for the proportion asymptomatic $p_{a}$. However, the fortran programs below could facilitate such a sensitivity analysis if there was more knowledge about the range of plausible values.

The calculations and simulations also assume discrete time steps and a different analysis will be required to account for a more realistic model in continuous time. ${ }^{[9]}$ However, one might reasonably hope that more realistic simulations in continuous time will yield fairly similar results to these simpler simulations - but this would need to be checked. 
There are some assumptions that are too pessimistic. Some assumptions that are likely too conservative have been mentioned above, but there are others. For example, many asymptomatic cases may be detected by contact tracing. There is also implicitly some assumptions that may be too optimistic. For example, it is assumed that there are not cases of prolonged infectious periods or human carrier states or animal reservoirs of the virus in the region. Nevertheless this study gives cause for optimism about the length of lockdown required for any region attempting to eliminate this disease.

\section{Acknowledgements}

I thank Profs Shijit Kumar and James McCaw of Melbourne University for their helpful comments.

\section{References}

[1] Galvani A, May RM. Dimensions of Superspreading. Nature 2005:438;pp293-295

[2] Lloyd-Smith JO, Schreiber SJ, Kopp PE, et al. Superspreading and the effect of individual variation on disease emergence. Nature. 2005; 438(7066): 355359 .

[3] Endo A, Abbott S, Kucharski AJ et al. Estimating the overdispersion in COVID-19 transmission using outbreak sizes outside China. Wellcome Open Research, awaiting peer review. Accessed 19/4/2020

[4] Kucharski AJ, Russel TW, Diamond C, Liu Y, et al. Early dynamics of transmission and control of COVID-19: a mathematical modelling study. Lancet, Infectious Diseases, Volume 20, (5), pp553-558, May 01, 2020

[5] Qifang Bi, Yongshen Wu, Shujiang Mei et al. Epidemiology and transmission of COVID-19 in 391 cases and 1286 of their close contacts 
in Shenzhen, China: a retrospective cohort study. Lancet, Infectious Diseases Published:April 27, 2020DOI:https://doi.org/10.1016/S14733099(20)30287-5 accessed 8/5/2020.

[6] Gongalsky M. Early detection of superspreaders by mass group pool testing can mitigate COVID-19 pandemic MedRXiv doi: https://doi.org/10.1101/2020.04.22.20076166, accessed 28/4/2020

[7] Feller W. Probability Theory and its Applications. Vol 1 P275, $2^{\text {nd }}$ edition, Wiley, 1961

[8] Ferguson NM, Laydon D, Nedjati-Gilani G et al. Impact of nonpharmaceutical interventions (NPIs) to reduce COVID-19 mortality and healthcare demand. Imperial College London (16-03-2020), doi: https://doi.org/10.25561/77482.

[9] Kimmel M, Axelrod D. Branching Processe in Biology. Interdisciplinary applied mathematics, Vol 19, Springer, 2001

\section{Attachment 1}

It is assumed that the number of asymptomatic cases is given by a geometric distribution, so that the probability that there are $\mathrm{k}$ asymptomatic cases is $\left(1-p_{a}\right) p_{a}^{k}, \quad k=0,1,2, \ldots$

The probability $p_{T 0}$, that none of these cases gives rise to ongoing transmission is found by summing the probability over all possible values of $k$ that there are $k$ such cases and that transmission from all such cases goes extinct with probability $p_{e}^{k}$

this gives $p_{T 0}=\sum_{k=0}^{\infty}\left(1-p_{a}\right) p_{a}^{k} p_{e}^{k}=\frac{\left(1-p_{a}\right)}{1-p_{a} p_{e}}$

If we include the conservative assumption that before diagnosis, the last case diagnosed had definitely seeded a single case, we have the result $p_{T 0}=\frac{\left(1-p_{a}\right) p_{e}}{1-p_{a} p_{e}}$ 


\section{Attachment 2}

Fortran program using Newton's method to calculate the probability of extinction of the branching process for various probability distributions

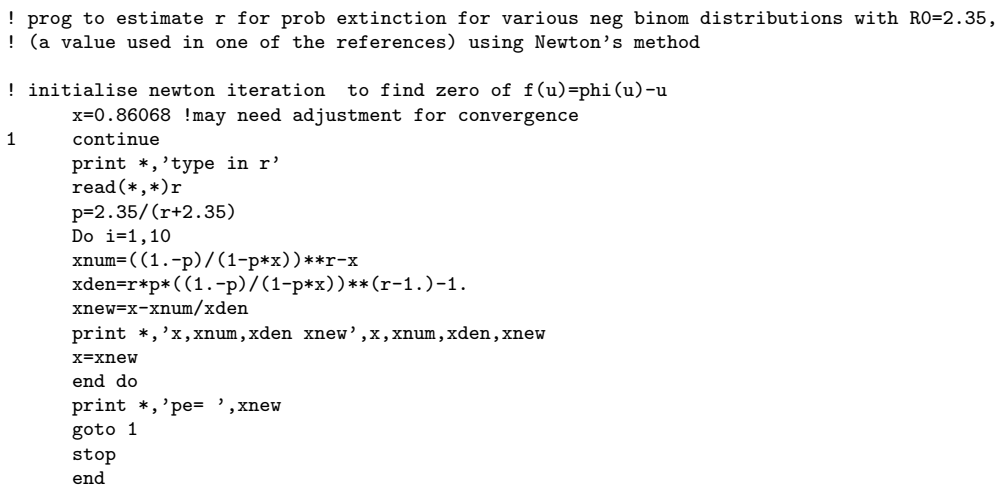

The fortran program below calculates by simulation, the probability of elimination when there are two incubation periods in which, under "lockdown" conditions, number of secondary cases are determined by a negative binomial distribution, with $R_{0}$ reduced from 2.5 to 0.8 , but with $r$ unchanged unchanged. Since this negative binomial is used repeatedly in the simulation, it is useful to calculate its cumulative distribution using a separate program. A few other very short, almost trivial, programs are also used in this project. They are not copied here but are available on request to the author.

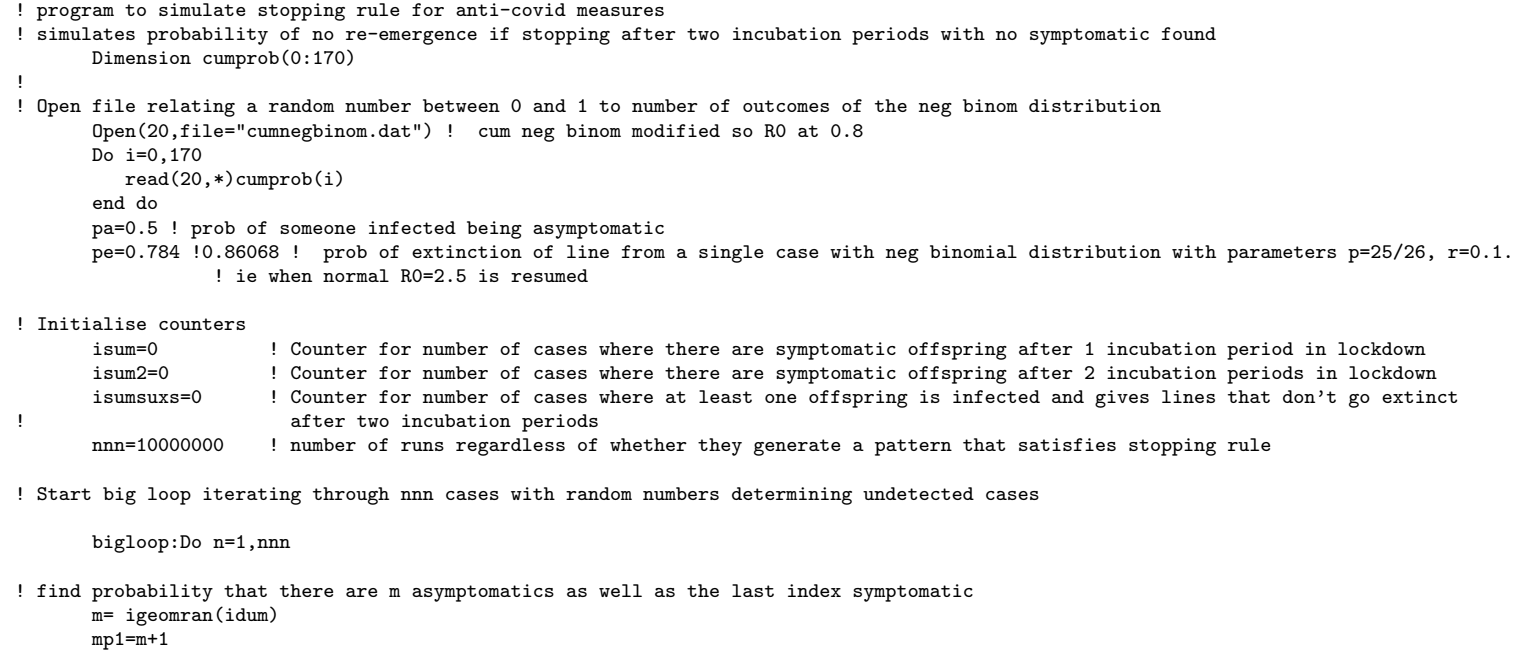


medRxiv preprint doi: https://doi.org/10.1101/2020.04.19.20071761; this version posted May 15, 2020. The copyright holder for this preprint (which was not certified by peer review) is the author/funder, who has granted medRxiv a license to display the preprint in perpetuity.

It is made available under a CC-BY-NC 4.0 International license .

! Count offspring of index case plus offspring of all asymptomatics

call totoffcalc(mp1, cumprob, ioffspring) ! ioffspring is a counter for total number of offspring from $\mathrm{m}+1$ neg binom distributions

print *, 'mp1 ioffspring', mp1, ioffspring

I Now need to find probability that all offspring are asymptomatic and if so the probability that all lines of descent from these asymptomatics ! end in extinction.

! Go back to beginning without counting stopping rule as a failure if still >=1 symptomatics present

$\mathrm{w}=\mathrm{ran} 3$ (idum)

if(w>pa**ioffspring)then !This gives a probability 1- pa`ioffspring so it is the probability there are ioffspring and some are symptomatic.

isum=isum +1

cycle bigloop

!Need to count such cases so that we can deduct them from our nnn simulations

!as they do not meet the stopping rule protocol

else

call totoffcalc(ioffspring, cumprob, ioffsprng2)

$\mathrm{w}=\mathrm{ran} 3($ idum $)$

if (w>pa**ioff sprng2) then

isum=isum +1

cycle bigloop

!This gives a probability 1- pa^ioffsprng2 so it is the probability there are ioffsprng2

end if

with some symptomatic. Need to count such cas

$\mathrm{w}=\mathrm{ran} 3$ (idum)

if $(\mathrm{w}<$ pe**ioffsprng2) then $\quad$ ! we have met the stopping rule protocol and all remaining lines go extinct

end if

end if umsuxs=i sumsuxs +1

!! print $*$, 'ptotextinct, w, is, isuxs ', ptotextinct, w, isum, isumsuxs

end do bigloop

probsuccess=real (isumsuxs) /real (nnn-isum)

print *, 'isumsuxs, isum, probsuccess', isumsuxs, isum, probsuccess

stop

subroutine totoffcalc(mp1, cumprob, ioffspring)

Dimension cumprob $(0: 170)$

iof fspring $=0$

Do $j=1$, mp1

$\mathrm{v}=\mathrm{ran} 3(\mathrm{~d}$ dum)

Do $i=0,170$

Do $i=0,170$

print $*,{ }^{\prime}, v, \operatorname{cump}(0), \operatorname{cump}(i)$ ', $i, v, \operatorname{cumprob}(0), \operatorname{cumprob}(i)$

exit

end if

end do

$\mathrm{k}=\mathrm{i}$

print $*,{ }^{\prime} i, k=\prime, i, k$

ioffspring=ioffspring $+k$

end do

return

end 